\title{
Kinematics Modeling and Simulation Analysis of Wheeled Mobile Robot in Round Pipe
}

\author{
Yuxia Zhao ${ }^{1,2, a}$, Yiqing Hua ${ }^{1, b}$ and Jiejian $\mathrm{Di}^{1, \mathrm{c}}$ \\ ${ }^{1}$ Department of Mechanical and Material Engineering , North China University of Technology, \\ Beijing, 100144 , China; \\ ${ }^{2}$ School of Mechanical Engineering, University of Science and Technology Beijing; Beijing,China \\ azhaoyx@ncut.edu.cn, ${ }^{b} 2424183492 @ q q . c o m,{ }^{c}$ dijiejian@ncut.edu.cn
}

Keywords: Wheeled motive robot; ADAMS, Kinematics modeling, Simulation analysis

Abstract. Micro in-pipe robot is a kind of mobile robot, which is suitable for small caliber pipe. The mobile mechanism is one of the important research contents in the field of robot research. ADAMS software is used to simulate the 3D solid motion of the six wheeled robot, and the kinematic model of the constrained micro robot in pipeline is established. The paper discussed the 3D solid motive simulation of six-wheeled robot, and the simulation result provides an useful method to study the shape and size of robot workspace. Through the centroid of the displacement, velocity and acceleration curves, spring force and driving torque curve analysis, it is proved that the movement of the six wheeled robot in the straight pipe has the basic motion stability and feasibility.

\section{Introduction}

Robot technology is a rapid development of high and new technology, it is the machinery, electronics, automation and integration of information processing technology. With the development of science and technology of microelectronics mechanical systems, micro robot technology arises at the historic moment. Micro robot technology becomes an important direction of MEMS research and content. Micro robot has a very wide range of applications, such as used for the transportation, medical, military, underwater operation, aerospace, extremely dangerous places (such as nuclear power station, fire, earthquake, etc.), industrial production, entertainment, etc. Up to now, a number of researchers have developed robots that can drive inside pipelines. In-pipe robots can be classified into several elementary forms according to the locomotion mechanism as shown in Fig. 1. Most of them have been designed based on specific tasks.

As shown in Fig. 1(a), for example, the pig is one of the most well known commercial one that is passively driven by fluid pressure inside oil pipelines and employed for the inspection of pipelines with large diameters[1]. The wheel type illustrated in Fig. 1(b) is similar to the plain mobile robot and a number of commercialized can be found [2]-[4]. Fig. 1(c) shows the crawler type robot with caterpillars instead of wheels [5]. As shown in Fig. 1(d), the wall press type denotes the robot with flexible mechanism for pressing the wall whatever means that has advantage in climbing vertical pipelines. As depicted in Fig. 1(e), walking type possesses articulated legs and it can produce various motions [6]. The inchworm type given in Fig. 1(f) is usually being developed for pipelines with very small diameter [7], [8]. Screw type one takes the motion of a screw when it advanced in the pipelines as depicted in Fig. 1(g) [9],. Most of in-pipe robots take the mechanism derived from one of those basic mechanisms or their combinations.

Generally speaking, the inside of pipelines has special conditions of narrow space and dimensional shapes, even with more complex irregular shapes. such features limit the size and shape of a robot and makes it difficult for the robot to move smoothly. But there are a wide variety of pipelines which is applied to versatile fields and should be inspected and maintained to ensure the safety and integrity. Thus the research on such robot is still more attractive and prosperous. 


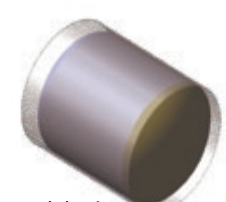

(a) pig type

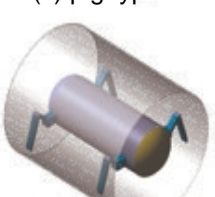

(e) walking type

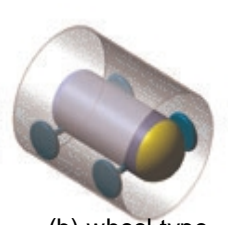

(b) wheel type

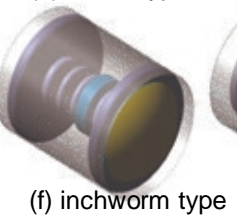

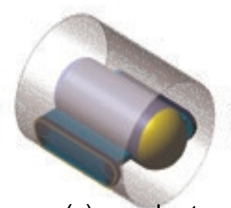

(c) crawler type

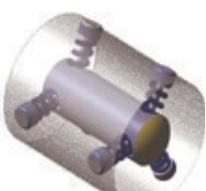

(d) wall press type
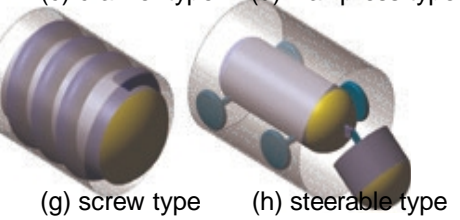

Fig. 1 Classification of in-pipe robots

Due to the limit of small pipe work environment, wheeled and crawling robot are mainly adopted in the actual research. In practical applications, the wheeled robot is the best way to move. Wheeled robot can be used in pipe, the diameter from a few inches to several feet. Wheeled robot has good mobility, high transmission efficiency. In addition, the wheeled robot can be easily miniaturized, which has simple driving mechanism and short transmission chain.

The paper discusses the 3D solid motive simulation of six-wheeled robot based on ADAMS software, and the simulation result provides an useful method to study the shape and size of robot workspace. The paper introduces the wheel mechanism design of in-pipe robot, establishes the kinematics model, and carries on the kinematics analysis for the model. This mobile robot mechanism has many advantages, such as compact structure, high efficiency, installation convenience, high reliability and low cost.

\section{ADAMS kinematics equation}

There exists motion pair connection between components and ground(or components), establishing the kinematics model based on ADAMS software. These motion pair can be expressed in generalized coordinates system as the algebraic equation, here only integrity constraints are considered. The number of constraint equations for the motion pair is set as $n h$, and then the system generalized coordinate vector is used to represent the kinematic constraint equations, the kinematic constraint equations are as follows.

$$
\phi^{k}(q)=\left[\phi_{1}^{k}(q), \phi_{2}^{k}(q), \ldots, \phi_{n h}^{k}(q)\right]^{T}=0
$$

Considering kinematics analysis, in order to make the system have certain movement, the degree of freedom of the system is zero. The driving equation of equal degree of freedom $(n c-n h)$ is applied to the system.

$$
\phi^{D}(q, t)=0
$$

In general, the drive constraint is a function of the generalized coordinates and time of the system. Driven constraints within the set of its set and the kinematic constraints must be independent and compatible, In this condition, the kinematics of the drive system is determined, and the motion is determined.

Drive constraints can be represented as.

$$
\phi(q, t)=\left[\begin{array}{l}
\phi^{K}(q, t) \\
\phi^{D}(q, t)
\end{array}\right]=0
$$

$n c$ nonlinear equations with $n c$ generalized coordinates are formed, the position equation of the system is formed.

By taking the derivative of the formula (3), velocity constraint equations are achieved as follows.

$$
\begin{aligned}
& \dot{\phi}(q, \dot{q, t})=\phi_{q}(q, t) \dot{q}+\phi_{t}(q, t)=0 \\
& v=-\phi_{t}(q, t)
\end{aligned}
$$

$\mathrm{V}$ is defined in (5), and thenThe velocity equation is defined in (6) 


$$
\dot{\phi}(q, \dot{q}, t)=\phi_{q}(q, t) \dot{q}-v=0
$$

By taking the derivative of the formula (6), acceleration equations can be achieved as follows.

$$
\ddot{\phi}(q, \dot{q}, \ddot{q}, t)=\phi_{q}(q, t) \ddot{q}+\left(\phi_{q}(q, t) \dot{q}\right)_{q} \dot{q}+2 \phi_{q t}(q, t) \dot{q}+\phi_{t t}(q, t)=0
$$

If $\eta=-\left(\phi_{q} \dot{q}\right)_{q} \dot{q}-2 \phi_{q t} \dot{q}-\phi_{t t}$, and then acceleration equations are as follows.

$$
\ddot{\phi}(q, \ddot{q}, \ddot{q}, t)=\phi_{q}(q, t) \ddot{q}-\eta(q, \dot{q}, t)=0
$$

Matrix $_{\phi_{q}}$ is Jacobi matrix. If the dimension of the matrix $\phi$ is $m$, the dimension of the matrix $q$ is $n$ , and then the dimension of the matrix $\phi_{q}$ is $m \times n, \phi_{q}$ is defined as ${\left.\left(\phi_{q}\right)_{(i, j)}\right)}_{\partial \phi_{i}} / \partial q_{j}$. In here $\phi_{q}$ is matrix $n c^{*} n c$ ( $n h$ is the number of kinematic constraint , $n c-n h$ is the number of driving constraint , $n c$ is the number of generalized coordinates ) .

\section{Modeling and simulation}

\section{Geometry Modeling.}

Stop block creation. By clicking ${ }^{\circ}$, inputting length, radius, starting point, end point, renaming, stop block modeling is finished.

Intermediate shaft creation. By clicking $O$, inputting length, radius, starting point, end point, stop block left cylinder modeling is finished. Creating the right cylinder is created similarly. By clicking

button, choosing stop block and left cylinder, Boolean addition operation is finished as a PART; By clicking again, choosing the PART and right cylinder, Boolean addition operation is finished as a intermediate shaft.

Sliders creation. By clicking $C$, creating circular ring, clicking 4 button, cylinder modeling of left slider is finished. Creating the right cylinder is created similarly. By clicking 9 , choosing Add to part option, inputting length, width and height, choosing left slider as Part, the starting point and end point, three connections are created successfully. And then the rotation and other steps, the geometric modeling of the two sliders is completed.

Spring creation. By clicking 홍. , selecting corresponding parameters, two springs modeling is finished.

Complete frame creation. By clicking 6 , circular ring is created; clicking 4 , cylinder modeling of left complete frame is finished. The right cylinder is created similarly. And then the rotation and other steps, the geometric modeling of the two complete frames is completed. Choosing the intermediate and right complete frame, Boolean addition operation is finished as a Part.

Connection Creation. By clicking $\mathcal{Q}, 15$ connections are created in turn.

Wheel set creation. By choosing 0 , , 6 wheel sets are finished.

The geometric modeling of the whole moving part of the robot is completed, as shown in Fig.2. 


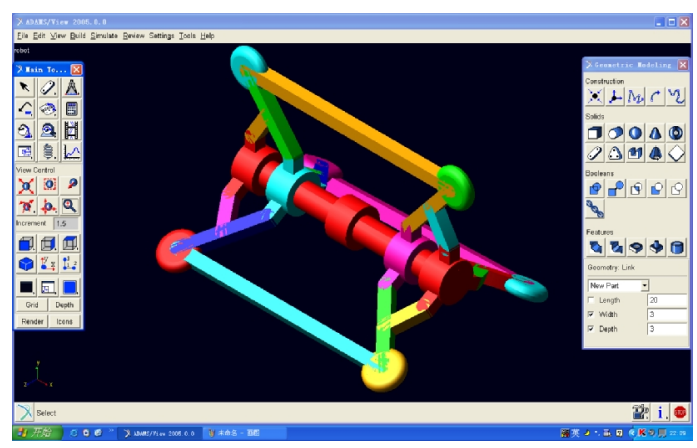

Fig.2 the geometric modeling of the robot

\section{Constraint addition.}

Mobile side creation between slider and intermediate. By choosing $\$$, selecting $2 b o d-110 c$ and Pick Feature options, selecting the left slider and intermediate shaft in turn, and then click the left slider, select the right or left to the direction of movement, the sliding pair of left slider and intermediate shaft is added. The sliding pair of right slider and intermediate shaft is finished.

Revolute pair creation. By choosing , selecting 2 bod-1loc and Normal to grid options ,selecting the left slider and connection 1 in turn, and then click Marker point of connection 1, the revolute pair of them is created. In the same way, the rotating pairs are connected with connection 1 and 2 , connection 2 and left complete frame, connection 1 and 3, connection 1 and wheel 1. Due to the use of the symmetrical mechanism, so the right part of the rotary side can refer to the left half done. To this point, all the constraints are added to the mobile ontology. The geometric model with constraint is added as shown in Fig.3.

\section{Simulation environment.}

Set working grid. On the setting menu, select the Grid Working command, select Global YZ option in Set Orientation, set the work grid to $Y Z$ direction, select pick option inSet Location, Choose the Marker point of the end face of the left complete frame as the coordinate origin.

Straight pipe creation. By choosing 2 , in the Length input 100, Radius respectively 29 and 31 , 2 cylinders are created. Selecting $[9]$, in order to select a large cylinder and a small cylinder, the above 2 cylinders are operated by Boolean subtraction to complete the creation of the pipeline. Selecting [8], select the 1 Location option, select the left Marker point of the pipe as the constraint point, create the locking constraint between the pipe and the earth.

Add contact force. First, select , create Marker points on top point of the wheel 1.Selecting , selecting Solid to Solid option, click the right mouse button in the Solid J and Solid I columns, respectively, select Pick option in Contact_solid, Select wheels 1 and pipes in turn, select Coulomb option in Fraction Force, Enter 0.3 in the Static Coefficient bar, enter 0.1 in Dynamic Coefficient bar , enter 10 in Stication Transition Vel.bar , enter 10 in Fraction Transition Vel.bar , Complete the contact between the wheel 1 and the pipe. The other 5 wheels in accordance with the above operation one by one are completed. 
The model of the robot to create the simulation environment in ADAMS is shown in Fig.4.

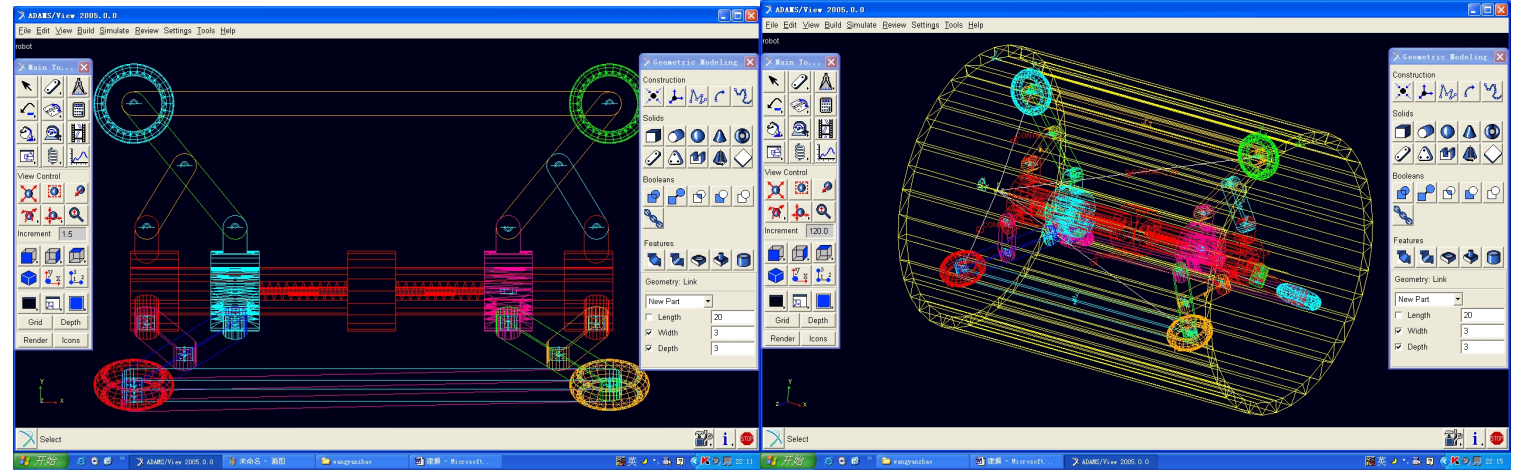

Fig.3 the geometric model with constraint

Fig.4 the model of the robot to create the simulation environment

Drive addition. Selecting (2) , enter 90 in Speed bar, select the rotation constraint between the connection 1 and the wheel 1 as the driving point to complete the drive.

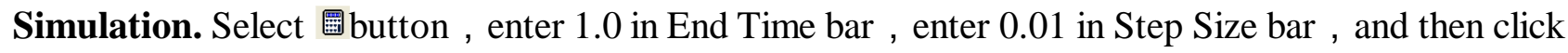
simulation button, software began to simulate the prototype model of the robot.

\section{Post simulation processing.}

Start ADAMS/PostProcessor, in the main toolbox, selecthn, display ADAMS/PostProcessor program interface.

set the window layout, in the main toolbar, select $\mathrm{m}$ min the right key, set 6 windows.

reproduce the simulation process.

Activate the upper left corner of the screen window, put the mouse on the window, click on the right mouse button to open the pop-up menu.

Select Load Animation command, call ADAMS/View simulation results, you can see the robot mechanism which has been transferred to the screen, as shown in Fig.5.

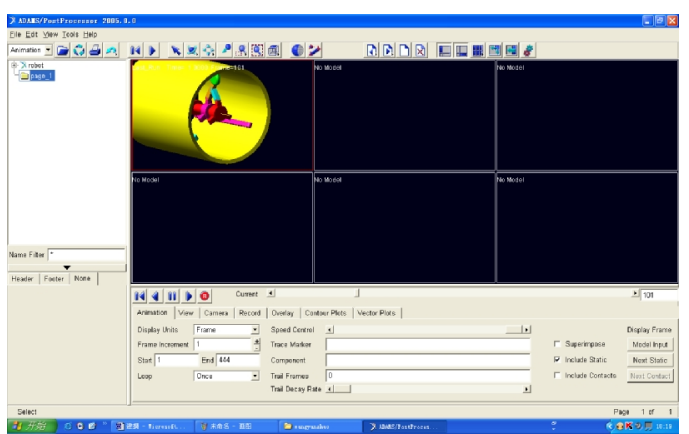

Fig.5 call the robot interface

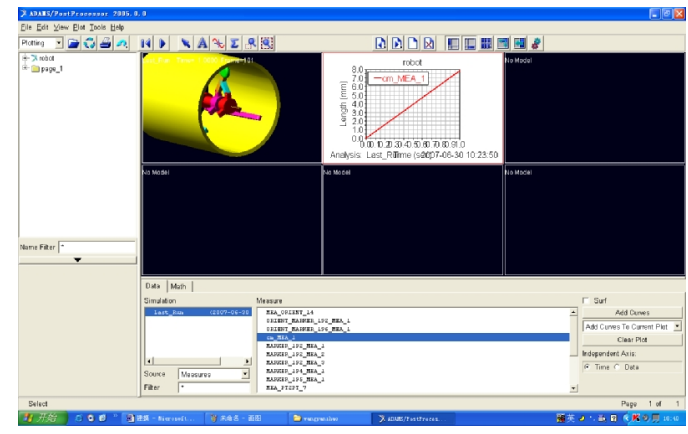

Fig.6 The centroid displacement curve of the interface

Draw the curve of the center of mass displacement, click on the right mouse button in the second window, select Load Plot command, and then select CM_MEA_1 in Measure , click add curves button, the centroid displacement curve of the interface after the call as shown in Fig.6.

The center of mass velocity and acceleration curve, spring force curve and drive torque curve after the call are as shown in Fig.7. 


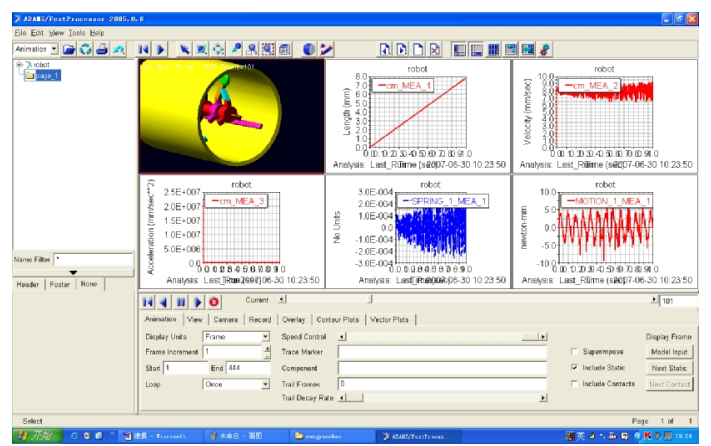

Fig.7 The center of mass velocity and acceleration curve, spring force curve and drive torque curve

\section{Simulation analysis.}

The displacement curve of the mass center shows that the moving body of the robot moves in a horizontal straight line, and the results are basically consistent with the expected results. Because of the influence of pipe wall friction, the wheel mechanism of gravity and impact, all these factors resulted in the speed around the small numerical range of shocks. The spring force also changed near the equilibrium position, the driving torque was also close to the sine curve in the case of some special moment value.

Because the robot is mainly rely on the 2 spring tension so that the wheels pressed on the pipe wall, and the inner wall of the wheel and the pipeline is generated enough pressure and friction, and of course the related the friction coefficient of the wheels and the inner wall of pipeline. When driving on the wheel, because of the existence of friction, the wheel can be rolled along the inner wall of the pipeline, and the entire body can move forward. Therefore, when the spring force exists for wheels and wall pipes with collision and oscillation are affected by gravity, the contact degree of the wheels and the inner wall of pipeline changes, resulting in the change of friction, and then produces velocity small shocks.

Through the centroid of the displacement, velocity and acceleration curves, spring force and driving torque curve analysis, it is proved that the movement of the six wheeled robot in the straight pipe has the basic motion stability and feasibility.

\section{Conclusions}

In this paper, ADAMS software is used to accomplish the 3D modeling and Simulation of micro wheeled robot. In the process of modeling and simulation, many difficulties have been encountered, and many valuable experiences have been gained.

In the parameter settings, complete generation of variable is very difficult, later found that the original parameters of the required parameters are based on some basic parameters, complex parameters are generated by the function editor on some of the basic parameters of the function.

In the process of geometric modeling, due to the lack of attention to the respective components of their own coordinate system, so that the simulation of the late encountered a lot of difficulties. For example, to measure a point of displacement or velocity, especially in a coordinate axis displacement or velocity, it is necessary to coordinate rotation to the right direction of the point, otherwise the curve will get wrong.

Add constraints, must ensure that the correct orientation and position constraints, selecting components must be in the order of first selection of active re follower, or will cause interference and error. Grid Working must not be displayed in the normal view to ensure the correct direction of the constraint. When adding compound pair, we should pay attention to select the active pair first, and then choose the order of the slave pair.

Modeling and Simulation of wheeled mobile robot in pipe micro environment in the analysis of the mechanical structure design of the ventilation pipe cleaning robot, natural gas pipeline inspection robot, sewage pipe inspection robot pipeline robot has the reference significance, which is the basis of a kind of mobile robot motion analysis and control. The research lays the foundation for the establishment of a new pipeline robot model.. 


\section{Acknowledgements}

This work was financially supported by the Beijing Municipal Board of education on the face of the project (KM201610009006).

\section{References}

[1] J. Okamoto Jr, J. C. Adamowski, M. S. G. Tsuzuki, F. Buiochi, C. S. Camerini, "Autonomous System For Oil Pipelines Inspection”, in Mechatronics, Vol. 9, pp. 731-743, 1999.

[2] T. Okada and T. Kanade, "A Three-Wheeled Self-Adjusting Vehicle in a Pipe", in Int. J. of Robotics Research ,Vol. 6,No. 4, pp. 60-75, 1987.

[3] T. Okada and T. Sanemori, "MOGRER: A Vehicle Study and Realization for In-Pipe Inspection Tasks," in IEEE J. of Robotics and Automation, Vol. 3, No. 6, pp. 573-582, 1987.

[4] S. Hirose, H. Ohno, T. Mitsui, and K. Suyama, "Design of In-pipe Inspection Vehicles for $\varphi 25$, ५50, 150 pipes", in Proc. Of IEEE Int. Conf. on Robotics and Automation, pp. 2309-2314, 1999.

[5] H. T. Roman, B. A. Pellegrino, W. R. Sigrist, "Pipe crawling inspection robots: an overview" , in IEEE Trans of Energy Conversion,pp. 576-583, 1993.

[6] W. Neubauer, "A Spider-Like Robot that Climbs Vertically in Ducts or Pipes," in IEEE/RSJ Int. Conf. on Intelligent Robots and Systems(IROS), pp. 1178-1185, 1994.

[7] T. Fukuda, H. Hosokai, M. Uemura, "Rubber gas actuator driven by hydrogen storage alloy for in-pipe inspection mobile robot with flexible structure", in IEEE Int. Conf. on Robotics and Automation(ICRA), Vol. 3, pp. 1847-1852, 1989.

[8] C. Anthierens, A. Ciftci, and M. Betemps, "Design of an electro pneumatic micro robot for in-pipe inspection", in IEEE Int. Symposium on Industrial Electronics(ISIE), Vol. 2, pp. 968-972, 1999.

[9] Hayashi I, Iwatsuki N, Iwashina S.The Running characteristics of a screw principle microrobot in a small bent

pipe [A].IEEE Sixth International Symposium on Micro Machine and Human Science[C]. Nagoya, Japan,

1995:225-228.

[10] Campion G, Bastin G, D Andrea-Novel B. Structural properties a classification of kinematics and dynamic models of wheeled mobile robots[J]. IEEE Transactions on Robotics and Automation, 1996, 12(1): 47-62.

[11] Lamon P, Krebs A, Lauria M, etal. Wheel torque control for rough terrain rover[A]. Proceedings of the 2004

IEEE International Conference on Robotics and Automation[C]. Piscataway, USA: IEEE, 2004, $4682-4687$. 